\title{
Validação de um instrumento para caracterização e verificação de fatores associados ao desempenho de corredores de rua
}

\author{
Validation of an instrument to characterization and identification of factors related do road \\ runners performance
}

\author{
M. Thuany ${ }^{1,2,3^{*}} ;$ T. N. Gomes ${ }^{1,3} ;$ M. B. de Almeida ${ }^{1,2}$ \\ ${ }^{I}$ Programa de Pós Graduação em Educação Física, Departamento de Educação Física, Universidade Federal de \\ Sergipel PPGEF - UFS, 49100-000, São Cristóvão - SE, Brasil. \\ ${ }^{2}$ Laboratório de estudo e pesquisa em performance no exercício e no esporte (L'esporte), Departamento de Educação \\ Física, Universidade Federal de Sergipe, 49100-000, São Cristóvão-SE, Brasil. \\ ${ }^{3}$ Centro de investigação em Saúde, Atividade Física e Esporte (SAFE), Departamento de Educação Física, \\ Universidade Federal de Sergipe, 49100-000, São Cristóvão - SE, Brasil. \\ *mablinysantos@gmail.com
}

(Recebido em 10 de dezembro de 2019; aceito em 06 de março de 2020)

\begin{abstract}
Tem-se observado, nos últimos anos, um aumento substancial no número de praticantes de corrida de rua no Brasil, os quais apresentam perfis e propósitos variados - alguns na busca pela competição/rendimento, outros com propósito de melhora de aspectos relacionados à saúde e qualidade de vida. Nesse sentido, o presente trabalho visa validar um instrumento que permita a caracterização deste público e a verificação do papel desempenhado por fatores de diferentes níveis no desempenho da corrida de rua. A amostra foi composta por 241 indivíduos, de ambos os sexos, com média de idade de 36,1 $\pm 10,3$ anos, praticantes de corrida de rua no Brasil, dos quais 10\%, aleatoriamente selecionados, responderam o instrumento duas vezes, respeitando-se o intervalo de um mês entre os dois momentos de resposta. O instrumento passou por dois processos de análise de conteúdo, realizada por avaliadores, seguida pela análise de reprodutibilidade, realizada pelo Coeficiente de Correlação Intraclasse (ICC) e o Coeficiente Kappa, o que indicou alta reprodutibilidade nas respostas, exceto para a variável "bairro de residência". Assim, verifica-se que o presente instrumento pode ser utilizado na condução de pesquisas futuras, acrescendo os benefícios de sua aplicação no formato eletrônico, permitindo a aplicação em diferentes amostras, bem como a comparação de resultados entre sujeitos de diferentes locais, para além do fornecimento de informações que permitam a ampliação e compreensão acerca dos fatores associados ao desempenho na corrida de rua.
\end{abstract}

Palavras-chave: Corrida de rua, questionário, validação.

It has being observed, over the last years, a relevant increase in the number of road runners in Brazil, which have different profiles and purposes - some aiming performance, others aiming to improve their health and quality of life. So, the present study aims to validate an instrument able to characterize this and also to verify the role of factors from different levels on road running performance. The sample comprised 241 subjects, of both sexes, with mean age of $36.1 \pm 10.3$ years, practitioners of road running in Brazil, of which $10 \%$ answered the questionnaire twice, with one-month interval between the two answered moments. The instrument submitted to two content analysis processes, performed by experts, followed by the reproducibility analysis, performed by the Intraclass Correlation Coefficient (ICC) and the Kappa Coefficient, which indicated high reproducibility in the answered, except for the variable "neighbourhood of residence". Thus, it can be seen that the questionnaire can be used in future researches, with the benefit of the fact that it is applied in an electronic format, allowing its use in different samples, as well as, the comparison of results from subjects from different places, beyond to provide information for a better understanding about factors related to road running performance.

Keywords: road running, questionnaire, validation.

\section{INTRODUÇÃO}

Na última década verificou-se um aumento substancial no número de praticantes de corrida de rua no mundo e no Brasil [1-4]. Embora seja difícil quantificar esse público, dados não oficiais estimam que aproximadamente 4 milhões de brasileiros (distribuídos entre as cinco regiões do país) praticam a modalidade frequentemente, movimentando cerca de 3,2 bilhões de reais por ano na compra de acessórios esportivos e participação em eventos específicos [5]. 
Para grande parte do público, a procura pela prática da corrida de rua está associada à busca pela melhora dos aspectos relacionados à saúde e à qualidade de vida $[6,7]$. Não obstante, também é possível evidenciar que para uma parcela de seus praticantes, sobretudo aqueles com maior tempo na modalidade, o principal fator motivacional é a busca pelo rendimento esportivo [8]. Dessa forma, é possível sugerir que atualmente há dois tipos de corredores, os chamados 'corredores-amadores' e os 'corredores-recreacionais', que têm como lema "from competing to completing", enfatizando a participação contrariamente à competição, e demonstrando que entre um grupo aparentemente homogêneo, há grande variabilidade interpessoal no tocante aos objetivos com a prática [9].

Essa variância pode ser explicada pela diversidade de contextos onde os corredores transitam, sendo influenciados por variáveis relacionadas às características individuais (biológicos a comportamentais) e ambientais (treinamento, influência do treinador/família, oportunidade para prática) $[10,11]$. Tais fatores atuam como potencializadores no desempenho desses atletas, e modulam sua permanência na prática. Embora a mensuração das componentes biotipológicos seja de difícil acesso, análises relacionadas à influência do ambiente no desempenho tornam-se cruciais, visto que o ambiente construído se apresenta mais propício a intervenções e alterações [2].

Dessa forma, torna-se relevante identificar os fatores relacionados à prática em ambos os grupos de corredores de rua (sobretudo pelo uso de instrumentos mais acessíveis e de "baixo custo", como a utilização de questionários), permitindo sua caracterização e diferenciação. Contudo, ao nosso melhor conhecimento, a literatura científica ainda não apresenta um instrumento que permita identificar esse conjunto de informações de modo sintético e objetivo. Dessa forma, o presente estudo está centrado na proposição e validação de um questionário que permita a caracterização de corredores de rua, e análise de informações relacionadas ao ambiente desses corredores que possam estar relacionados à prática e ao desempenho na modalidade.

\section{MATERIAL E MÉTODOS}

\subsection{Amostra}

O presente estudo contou com a participação de 241 indivíduos, com média de idade de $36,1 \pm$ 10,3 anos, praticantes de corrida de rua no Brasil. A inclusão no estudo foi condicionada à idade mínima de 20 anos, sendo excluídos da análise os indivíduos que não respondessem a alguma das questões obrigatórias do questionário. Todos os voluntários foram informados sobre os objetivos e os procedimentos que seriam realizados, tendo, em seguida, consentido por escrito sua participação no estudo. O projeto teve aprovação prévia pelo Comitê de Ética em Pesquisa da Universidade Federal de Sergipe, sob o parecer $\mathrm{n}^{\mathrm{o}}$ 3.558.630. O questionário foi aplicado duas vezes para a referida amostra, respeitando-se o intervalo de um mês entre as abordagens, de modo que no segundo momento houve um retorno de $10 \%$ da amostra inicial.

\subsection{Procedimentos}

A construção do instrumento foi realizada baseando-se na literatura científica nacional, com abordagem específica sobre o perfil dos praticantes de corrida de rua e fatores relacionados ao desempenho esportivo [4, 12, 13], de modo que permitisse a obtenção de informações para caracterização do público e identificação dos fatores relacionados ao rendimento. Dessa forma, o mesmo foi dividido em seis blocos, organizados da seguinte forma:

- Dados de identificação do corredor - sexo e idade;

- Variáveis antropométricas - estatura $(\mathrm{cm})$ e massa muscular $(\mathrm{kg})$;

- Perfil sociodemográfico - local de residência (estado, capital ou não, e bairro); renda mensal; escolaridade; estado civil;

- Aspectos ambientais relacionados à prática de corrida - informações acerca da percepção dos corredores sobre fatores ambientais (condições climáticas e infraestrutura da cidade/estado);

- Variáveis do treinamento - frequência e volume de treinamento semanal $(\mathrm{km})$; sessões/dia; tempo de prática (em meses ou anos); ritmo de corrida $(\mathrm{min} / \mathrm{km})$; participação em prova nos últimos 12 meses; turno de treinamento; distância preferida $(5 \mathrm{~km}, 10 \mathrm{~km}, 21 \mathrm{~km}, 42 \mathrm{~km}$, outros); motivação para prática; participação em grupos de corrida; se possui ou não um treinador, bem como a influência desse no desempenho; 
- Ambiente familiar - composição familiar; existência de praticantes de corrida de rua na família; prática esportiva na infância bem como suporte parental para essa prática.

Posteriormente à construção do instrumento, realizou-se uma análise de conteúdo, para verificação sobre a pertinência das questões e coerência com o objetivo principal. O procedimento inicial foi realizado por três avaliadores independentes, dos quais dois têm experiência no que diz respeito à pesquisa científica, e o terceiro parecerista foi um profissional com atuação específica em corrida de rua. Nessa etapa, os itens que compõem o questionário foram divididos em blocos (apresentados anteriormente) e avaliados em uma escala de 0 a 4 , nos seguintes aspectos: "conteúdo", "objetividade", "clareza", "facilidade de leitura" e "compreensão do conteúdo", sendo considerado ' 0 ' quando o item não contemplasse de maneira alguma o critério a que se referia, e '4' se o item satisfizesse completamente o critério [14].

Após a realização de alterações sugeridas, o instrumento passou novamente por um processo de avaliação, realizada por cinco avaliadores, como propõe Lynn (1986) [15], sendo mantidos os três avaliadores iniciais e acrescendo outros dois, com expertise em pesquisa científica. A avaliação foi dividida em dois momentos: 1) Avaliação dos itens e 2) Avaliação dos domínios, sendo realizado em seguida uma análise de concordância entre os juízes, através do cálculo da taxa de concordância do comitê (número de participantes que concordaram/número total de participantes) x $100=\%$ de concordância). O valor mínimo para aceite de cada domínio foi $90 \%$, de modo que ao não ser atingido deveria haver uma reconsideração do referente domínio [16].

O procedimento para coleta dos dados foi feito mediante envio online (em redes sociais e contato de profissionais que atuam na área) do questionário "Caracterização do perfil e fatores associados ao desempenho em corrida de rua", entre os meses de setembro/outubro de 2019.

\subsection{Análise estatística}

A informação descritiva foi feita através da verificação de frequência (para as variáveis apresentadas em categorias) e média e desvio padrão (para as variáveis contínuas). A análise de reprodutibilidade para as variáveis contínuas foi realizada pelo Coeficiente de Correlação Intraclasse (CCI). O CCI (do inglês Intraclass Correlation Coeficient) ou coeficiente de reprodutibilidade $(\mathrm{R})$ é uma estimativa da fracção da variabilidade total de medidas, devido a variações entre os indivíduos, tendo sido adotado a seguinte classificação no presente estudo: pobre $(\mathrm{R}<0,4)$; satisfatória $(0,4 \leq \mathrm{R}<0,75)$; excelente $(\mathrm{R} \geq 0,75)$. O Coeficiente Kappa pode ser entendido com uma medida de associação que atesta a concordância entre duas medidas, tendo sido utilizado no presente estudo para os dados categóricos. A classificação dos itens avaliados seguiu a proposta por Landis e Koch (1977) [17], compreendendo: Kappa $<0,00=$ quase inexistente; 0,01 - 0,20 = pequena; $0,21-0,40=$ insatisfatória; $0,41-0,60=$ moderada; $0,61-0,80=$ substancial; 0,81 1,00 , quase perfeita. Os dados foram analisados por meio do programa SPSS, versão 24.0, sendo adotado $\mathrm{p}<0,05$ como nível de significância.

\section{RESULTADOS}

A análise por pares realizada inicialmente indicou a necessidade de alterações no bloco de itens relacionados ao treinamento e ambiente familiar (Tabela 1).

Tabela 1. Análise de conteúdo inicial dos tópicos apresentados no questionário "Caracterização do perfil e fatores associados ao desempenho em corrida de rua”.

\begin{tabular}{lccccc}
\hline & Conteúdo & Objetividade & Clareza & $\begin{array}{c}\text { Facilidade } \\
\text { de leitura }\end{array}$ & $\begin{array}{c}\text { Compreensão } \\
\text { do conteúdo }\end{array}$ \\
\hline Dados de identificação & 3,6 & 4 & 4 & 4 & 4 \\
Variáveis antropométricas & 4 & 4 & 4 & 4 & 4 \\
Perfil sociodemográfico & 3,6 & 3 & 3 & 2,6 & 4 \\
Aspectos ambientais & 4 & 4 & 3,3 & 3,6 & 3,3 \\
Variáveis do treinamento & 3,6 & 2,6 & 2,3 & 2,3 & 2,3 \\
Ambiente familiar & 3,3 & 4 & 3 & 3,6 & 3 \\
\hline
\end{tabular}


Em seguida, após realizados os ajustes necessários/sugeridos, o questionário passou pela segunda análise por avaliadores, levando em consideração os domínios e itens quanto à "compreensão" e "relevância" dos mesmos. Observou-se que o critério "compreensão" apresentou valores abaixo de $90 \%$ para as seguintes variáveis: estatura, estado, bairro, número de sessões/semanal, ritmo de corrida, composição familiar, prática esportiva na infância e suporte parental. Para o critério "relevância", os mesmos resultados foram observados para "bairro", "composição familiar", e "suporte parental", sendo cada questão analisada individualmente e reconsiderada para o instrumento final. No que diz respeito à análise dos domínios, não houve a necessidade de exclusão de itens, sendo feitos apenas correções nos referidos anteriormente (Tabela 2).

Tabela 2. Análise de conteúdo realizada pelos avaliadores, e reprodutibilidade da informação através do Coeficiente de Correlação Intraclasse e Kappa.

\begin{tabular}{|c|c|c|c|c|c|}
\hline Variável & Compreensão & Relevância & & & \\
\hline & & & CCI & $p$ & Alpha de Cronbach \\
\hline Peso & 100 & 100 & 0,99 & $<0,001$ & 0,99 \\
\hline Estatura & 80 & 100 & 0,99 & $<0,001$ & 0,99 \\
\hline Tempo de prática & 100 & 100 & 0,79 & $<0,001$ & 0,88 \\
\hline Quilometragem semanal & 100 & 100 & 0,89 & $<0,001$ & 0,94 \\
\hline \multirow[t]{2}{*}{ Ritmo médio de corrida } & 80 & 100 & 0,97 & $<0,001$ & 0,98 \\
\hline & & & Kappa & $p$ & \\
\hline Sexo & 100 & 100 & 1,00 & $<0,001$ & \\
\hline Estado & 80 & 100 & 1,00 & $<0,001$ & \\
\hline Capital & 100 & 100 & 1,00 & $<0,001$ & \\
\hline Bairro & 80 & 80 & 0,05 & 0,24 & \\
\hline Escolaridade & 100 & 100 & 0,87 & $<0,001$ & \\
\hline Estado Civil & 100 & 100 & 0,92 & $<0,001$ & \\
\hline Renda & 100 & 100 & 0,66 & $<0,001$ & \\
\hline $\begin{array}{l}\text { Percepção sobre a } \\
\text { influência do ambiente }\end{array}$ & 100 & 100 & 0,80 & $<0,001$ & \\
\hline Clima & 100 & 100 & 0,51 & $<0,001$ & \\
\hline $\begin{array}{l}\text { Tempo de prática (Até } 1 \\
\text { ano - Acima de } 1 \text { ano) }\end{array}$ & 100 & 100 & 0,21 & $<0,001$ & \\
\hline Motivação para a prática & 100 & 100 & 0,66 & $<0,001$ & \\
\hline Frequência/ semana & 100 & 100 & 0,63 & $<0,001$ & \\
\hline Sessões/ semana & 80 & 100 & 0,65 & $<0,001$ & \\
\hline Distância & 100 & 100 & 0,84 & $<0,001$ & \\
\hline $\begin{array}{l}\text { Participação em } \\
\text { competição }\end{array}$ & 100 & 100 & 0,37 & $<0,001$ & \\
\hline Prova & 100 & 100 & 0,16 & $<0,001$ & \\
\hline $\begin{array}{l}\text { Participação em grupo } \\
\text { de corrida }\end{array}$ & 100 & 100 & 0,75 & $<0,001$ & \\
\hline Planejamento anual & 100 & 100 & 0,59 & $<0,001$ & \\
\hline Treinador & 100 & 100 & 0,64 & $<0,001$ & \\
\hline $\begin{array}{l}\text { Importância do treinador } \\
\text { na prática }\end{array}$ & 100 & 100 & 0,37 & $<0,001$ & \\
\hline Classificação corredor & 100 & 100 & 0,62 & $<0,001$ & \\
\hline Influência para corrida & 100 & 100 & 0,68 & $<0,001$ & \\
\hline $\begin{array}{l}\text { Familiar praticante de } \\
\text { corrida }\end{array}$ & 100 & 100 & 0,83 & $<0,001$ & \\
\hline Composição familiar & 40 & 60 & 1,00 & $<0,001$ & \\
\hline $\begin{array}{l}\text { Prática esportiva na } \\
\text { infância }\end{array}$ & 80 & 100 & 0,62 & $<0,001$ & \\
\hline Suporte parental & 80 & 80 & 0,83 & $<0,001$ & \\
\hline
\end{tabular}

Nota: fórmula para análise dos itens pelos avaliadores, em "compreensão" e "relevância" = (número de participantes que concordaram/número total de participantes) x $100=\%$ de concordância). 
A amostra do presente estudo contou com a participação de 241 corredores de rua, distribuídos entre as cinco regiões do país, com média de idade de 36,3 $\pm 10,1$ anos, sendo $60,6 \%$ do sexo masculino. Verifica-se que grande parte do grupo possui mais de um ano de experiência na modalidade $(80,1 \%)$, refletindo (possivelmente) na sua autopercepção/classificação sobre o tipo de corredor (corredor amador), bem como frequência de treinamento semanal prevalente de " 3 a 4 treinos/semana" (49\% e 19,5\%, respectivamente). Relativamente aos fatores motivacionais, observou-se uma maior prevalência para as respostas busca pela "saúde e qualidade de vida" $(40,2 \%)$, bem como "prazer e bem-estar" $(22,4 \%)$.

A verificação da confiabilidade e reprodutibilidade, realizada através do CCI, demonstrou que todas as questões analisadas foram classificadas como excelente $(R \geq 0,75)$. Relativamente às questões categóricas, avaliadas por meio do Coeficiente de Kappa, a grande maioria dos itens apresentou valores significativos ( $\mathrm{p}<0,05)$ com concordância "substancial" a "quase perfeita", exceção feita para a variável "bairro", onde não foi verificada concordância. Além disso, as respostas referentes ao "tempo de prática", "participação em competição nos últimos 12 meses" e "importância do treinador no rendimento", apresentaram relação "insatisfatória" entre os dois momentos (Tabela 2).

\section{DISCUSSÃO}

$\mathrm{O}$ aumento do número de praticantes de corrida de rua tem sido evidenciado em diversos locais do mundo e do Brasil [4, 8, 12, 18], sobretudo entre os adultos, justificando a determinação da idade mínima de participação no presente estudo. Não obstante a realização de diferentes trabalhos, na perspectiva de compreender sobre o fenômeno em questão, bem como verificar o perfil de seus praticantes $[6,8]$.

Sabe-se que no campo das ciências do esporte há uma demanda relacionada à identificação dos fatores que contribuem para a maximização do desempenho [19], tornando-se relevante a construção e validação de instrumentos acessíveis para essa finalidade. No presente trabalho, o questionário foi pensado levando em consideração o aspecto multifatorial associado à performance e, para tanto, buscou-se obter informações acerca de aspectos antropométricos, local de residência, bem como aspectos ambientais e climáticos da região, fatores socioeconômicos, suporte parental para prática esportiva na infância e variáveis do treinamento [10].

Na perspectiva de verificação da validade e confiabilidade do instrumento, foram realizados o CCI e Coeficiente Kappa. A validade, entendida como capacidade de um instrumento medir aquilo que se propõe [20], foi realizada inicialmente a partir da análise dos avaliadores (tabela 1), permitindo, a partir dos resultados obtidos a verificação da confiabilidade. Conceitualmente, confiabilidade refere-se à estabilidade das respostas em momentos distintos [21], dando segurança ao pesquisador sobre a reprodutibilidade da mesma. De modo geral, após análise inicial e reformulação do questionário, o CCI e coeficiente Kappa, indicaram alta reprodutibilidade nas respostas (exceto para a variável "bairro de residência"). Vale ressaltar que tal variável foi pensada para condução de análises de georreferenciação, que identifiquem a possível relação entre a performance (determinada através da variável "ritmo"), bairro de residência e locais de práticas de atividades físicas, bem como os fatores socioeconômicos e demográficos da região em questão. Visto que a resposta a essa questão não é mandatória, já que sua ausência não inviabiliza a conclusão do questionário por parte dos participantes do estudo, ausência de resposta à mesma não interfere na qualidade da informação obtida, visto que o instrumento foi pensado em blocos, por forma que o pesquisador tenha a liberdade de optar por utilizar o instrumento levando em consideração todos os blocos e itens, ou utilizar blocos e/ou itens específicos, sem haver, portanto, uma interdependência entre as questões.

A exemplo pode ser citada a utilização das variáveis antropométricas de forma isolada (estatura e peso) ou em conjunto para a determinação do índice de massa corporal (IMC) [22], e posterior associação com as variáveis do treinamento, como observado em estudos conduzidos anteriormente [23-25]. Além disso, análises também podem ser conduzidas de forma a estratificar por sexo e/ou idades, provas de preferência dos atletas $(5 \mathrm{~km}, 10 \mathrm{~km}, 21 \mathrm{~km}, 42 \mathrm{~km}$, outros) etc., permitindo inferir de modo pontual para grupos de interesse.

Relativamente aos fatores ambientais (climáticos e estruturais), há um consenso quanto à influência dessas variáveis no desempenho em provas de endurance [2, 26]. Portanto, levando em 
consideração esses aspectos e tendo em vista a diversidade existente entre as regiões/estados do Brasil, é possível entender que essas diferenças podem culminar em níveis de performance distintos entre os corredores. Além disso, torna-se crucial verificar a percepção dos corredores sobre a interferência desses fatores em sua prática.

Vale ressaltar que a aplicação online do instrumento possibilita que os estudos sejam conduzidos comparando-se cidades, regiões e estados dentro do país. Além disso, após as respostas obtidas, é possível ampliar as possibilidades de análise, levando em consideração informações do ambiente externo ao corredor de rua, e que podem ser obtidas a partir de fontes oficiais [tais como Índice de Desenvolvimento Humano (IDH) do bairro e/ou estado de residência, renda per capita, tamanho da população, locais para prática de atividades físico-esportivas, número de competições realizadas nas mediações do bairro e/ou estado], podendo associar essas informações ao desempenho ou demais variáveis de interesse. No que concerne ao desempenho [mensurado na corrida de rua através do ritmo de corrida $=$ tempo $(\min ) /$ distância $(\mathrm{km})]$ este pode ser obtido através da questão "ritmo de corrida", e tal informação pode ser confirmada a partir do confronto com as provas das quais os corredores relataram participação.

Não obstante o instrumento tenha sido pensado para sanar lacunas existentes no tocante ao problema levantando, o mesmo apresenta algumas limitações, das quais podem ser referidas àquelas associadas à utilização de questionários na obtenção de informações: dificuldade de controle sobre o retorno das respostas, possibilidade de não resposta às questões, e dificuldade de compreensão ou mal interpretação de questões por parte do participante (embora procedimentos de análise de conteúdo tenham sido conduzidos para amenizar esses problemas). Porém, há de se destacar a possibilidade de realização de estudos com grande alcance, possibilitando caracterização de grupos em diversos locais e contextos.

\section{CONCLUSÃO}

O questionário "Caracterização do perfil e fatores associados ao desempenho em corrida de rua" foi elaborado com a perspectiva de ser um instrumento que permita a caracterização de corredores de rua do Brasil e amplie as possibilidades de obtenção de informações acerca dos fatores que podem estar associados ao desempenho nesse público. Após as análises conduzidas, observa-se que o instrumento apresentou boa estabilidade, indicando que o mesmo pode ser utilizado na condução de novos estudos. A forma de aplicação do instrumento permite uma maior abrangência amostral, o que possibilita a realização de estudos em larga escala, bem como a realização de comparação entre perfis e fatores associados ao desempenho entre corredores de diferentes locais. Mais, os diferentes domínios pelos quais versam suas perguntas também permitem que, consoante o propósito dos trabalhos, os dados possam ser analisados estratificados por sexo, idade, tempo de prática, IMC, estados do país, para além de outras possibilidades. Acrescenta-se que um ponto salutar é a possiblidade de associar, às respostas do questionário, informações acerca de fatores externos ao indivíduo (variáveis relacionadas ao bairro e/ou estado: IDH, quantidade de locais para prática da modalidade, bem como número de provas, tamanho da população, condição socioeconômica), ampliando o entendimento sobre a influência dessas variáveis na performance.

\section{REFERÊNCIAS BIBLIOGRÁFICAS}

1. FPA. Demonstrativo de Corridas de Rua nos Últimos Anos no Estado de São Paulo 2017 [Available from: http://www.atletismofpa.org.br/source/Demonstrativo-de-Corridas-de-Rua-nos-Ultimos-Anos-noEstado-de-Sao-Paulo-2017.pdf.[Nov 2019].

2. Alda A, Pérez L, Sanz J. La percepción que los corredores kenianos tienen de sus actividades entrenamiento. CPD. 2014;14(2):99-110.

3. Roriz Ferreira V, Nunes Bento AP, Reis Silva M. Consumo aAlimentar, perfil antropométrico e conhecimentos em nutrição de corredores de rua. Rev Bras Med Esporte. 2015;21(6):457-61.

4. Salgado JVV. Análise do perfil de corredores de rua [Doctoral Thesis]. Campinas: Universidade Estadual de Campinas; 2016.89 p.

5. Sebrae. Tendências do mercado de corrida de rua 2018 [Available from: https://sebraeinteligenciasetorial.com.br/produtos/boletins-de-tendencia/tendencias-do-mercado-decorridas-de-rua/5b5a1605d0a9751800f2af49.[04 dez]. 
6. Balbinotti MAA, Gonçalves GHT, Klering RT, Wiethaeuper D, Balbinotti CAA. Perfis motivacionais de corredores de rua com diferentes tempos de prática. Rev Bras Ciênc Esporte. 2015;37(1):65-73, doi: 10.1016/j.rbce.2013.08.001.

7. Van Dyck D, Cardon G, de Bourdeaudhuij I, de Ridder L, Willem A. Who participates in running events? Socio-demographic characteristics, psychosocial factors and barriers as correlates of non-participationa pilot study in Belgium. Int $\mathbf{J}$ Environ Res Public Health. 2017;14(11):1315, doi: 10.3390/ijerph14111315.

8. Malchrowicz-Mośko E, Poczta J. Running as a form of therapy socio-psychological functions of mass running events for men and women. Int J Environ Res Public Health. 2018;15(10):2262, doi: 10.3390/ijerph15102262.

9. Bottenburg M, Scheerder J. Chances and challenges of the second wave of running for European atlhetics' Member Federation. 2006. 34 p.

10. Georgiades E, Klissouras V, Baulch J, Wang G, Pitsiladis Y. Why nature prevails over nurture in the making of the elite athlete. BMC Genomics. 2017;18(Suppl 8), doi: 10.1186/s12864-017-4190-8.

11. Espstein D. A genética do esporte: como a biologia determina a alta performance esportiva. 1 ed. Rio de Janeiro: Elsevier; 2014. 306 p.

12. Oliveira ET. Características e fatores associados dos corredores de rua de Aracaju [Master Thesis]. Aracaju: Universidade Federal de Sergipe; 2015.67 p.

13. Cardoso AF, Ferreira ES, Dos Santos LV. Perfil socioeconômico de praticantes de corrida de rua da cidade de Teresina - PI. Rev Bras Nutr Esportiva. 2018 Jan/Dez;12(75):862-66.

14. Varanda SS, Benites LC, Souza Neto SD. O processo de validação de instrumentos em uma pesquisa qualitativa em Educação Física. Motrividência. 2019;31(57):1-15, doi: 10.5007/2175-8042.2019e53877.

15. Lynn M. Determination and quantification of content validity. Nurs Res. 1986;35(6):382-85, doi: 10.1097/00006199-198611000-00017.

16. Coluci M, Alexandre N, Milani D. Construction of measurement instruments in the area of health. Cien Saude Colet. 2015 Mar;20(3):925-36, doi: 10.1590/1413-81232015203.04332013.

17. Landis R, Kochi G. The Measurement of Observer Agreement for Categorical Data. Biometrics. 1977 Mar;33(1):159-74.

18. Scheerder J, Borgers J, Breedveld K. Running across Europe: The rise and size of one of the largest sport markets. 2015. 298 p.

19. Kiss M, Bohme M, Mansoldo A, Regazzini E. Desempenho e Talento Esportivos. Rev Paulista Educ Fís. 2004 Ago;18:89-100.

20. Roberts P, Priest H, Traynor M. Reliability and validity in research. Nurs Stad. 2006;20(44):41-45, doi: 10.7748/ns2006.07.20.44.41.c6560.

21. Pittman J, Bakas T. Measurement and instrument design. J Wound Ostomy Continence Nurs. 2010 Nov/Dec;37(6):603-07. doi: 10.1097/WON.0b013e3181f90a60.

22. Oliveira MFM, Caputo F, Greco C, Denadai B. Aspectos relacionados com a otimização do treinamento aeróbio para o alto rendimento. Rev Bras Med Esporte. 2010;16(1):61-66.

23. Gomes-Molina J, Ogueta-Alday A, Camara J, Stickley C, Rodriguez-Marroyo J, Garcia-López J. Predictive Variables of Half-Marathon Performance for Male Runners. J Sports Sci Med. 2017;16(2):18794.

24. Rüst CA, Knechtle B, Knechtle P, Barandun U, Lepers R, Rosemann T. Predictor variables for a half marathon race time in recreational male runners. J Sports Med. 2011;2(2):113-9, doi: 10.2147/oajsm.s23027.

25. Pazin J, Duarte M, Poeta L, Gomes M. Recreational road runners: injuries, training, demographics and physical characteristics. Rev Bras Cineantropom Desempenho Hum. 2008;10(3):277-282, doi: 10.5007/1980-0037.2008v10n3p277

26. El Helou N, Tafflet M, Berthelot G, Tolaini J, Marc A, Guillaume M, et al. Impact of environmental parameters on marathon running performance. Plos One. 2012;7(5):e37407, doi: 10.1371/journal.pone.0037407. 